\title{
Applying Instructional Design Principles on Augmented Reality Cards for Computer Science Education
}

\author{
Josef Buchner ${ }^{\left({ }^{\infty}\right)}(\mathbb{1})$ and Michael Kerres (10) \\ Learning Lab, University of Duisburg-Essen, Essen, Germany \\ josef.buchner@uni-due.de
}

\begin{abstract}
In this article we describe Augmented Reality (AR) cards for computer science education that were created in the PCBuildAR project. From a technological point of view, we use marker-based AR for the cards so that students can learn and practice at any time with their smartphones. The instructional approach is based on the components content, construction and communication (3C model). The content ensures the acquisition of knowledge, which then is applied via problem-based learning activities (construction). Communication not only takes place between learners, but also with teachers.

All materials will be available as open educational resources after the project is completed.
\end{abstract}

Keywords: Augmented Reality - Computer science education · Instructional design $\cdot 3 \mathrm{C}$ model

\section{Introduction}

How to solve technical problems is a subarea of computer science education in schools. Therefore, the physical components are required to un- and rebuild the computer. However, these are not available everywhere in sufficient quantity [1]. The PCBuildAR project $^{1}$ wants to address this lack and develops paper-based cards with $2 \mathrm{D}$ representations of the components of a computer system, which are enriched with virtual information using Augmented Reality (AR). Our focus is on the final use for learning of the AR cards. Our instructional approach is based on the three components of the $3 \mathrm{C}$ model [2], which we combine with the potential of AR technology.

\section{The 3C Model}

According to [2], each learning environment consists of three components in different proportions: content, construction, communication.

The content component offers learners instructionally prepared information that is designed for the learning objective. The content can be prepared in various forms, such

\footnotetext{
$\overline{1}$ https://www.pcbuild-ar.com.

(C) The Author(s) 2020

C. Alario-Hoyos et al. (Eds.): EC-TEL 2020, LNCS 12315, pp. 477-481, 2020.

https://doi.org/10.1007/978-3-030-57717-9_48
} 
as a lecture by a teacher, texts, videos, audios or animations. The content component aims at imparting declarative knowledge which is needed as a prerequisite for the processing of learning tasks of the other two components.

The construction component encourages learners to engage in learning activities that fits the learning objective. After successful completion of these, there are visible results, e.g. in the form of artifacts or products. The learning tasks can be designed both for individual and cooperative learning. Tasks are useful if the knowledge to be acquired through the learning environment is also to be applied, i.e. procedural knowledge is also part of the learning objective.

Communication enables learners to interact socially with other learners and/or teachers/mentors. This component creates opportunities to deepen knowledge in exchange with others, opens up opportunities for discussion and trains key competences such as conversation skills and the formulation of personal points of view.

The proportions of the respective components depend on the learning objective. For example, it is quite possible that a learning environment consists to a large extent of the components content and construction, while the communication component only makes up a small part or perhaps even do not occur at all [2].

\section{AR Cards}

AR cards represent a variation of AR-supported learning that can be used in different contexts [3]. AR cards have already been tested for learning English vocabulary [4], for collaborative work [5] and for the promotion of computational thinking skills [6]. From a technological point of view, such cards belong to the type of marker-based AR systems, since the superimposition of reality around virtual artifacts is done by the recognition of an image. Reality and virtuality are then displayed simultaneously, interactions are possible in real time and the virtual objects are aligned with the real environment [7].

As shown in $[4,6]$, AR cards can be used with mobile devices, e.g. tablet or smartphone.

\section{The 3C Applied to AR Cards}

In the PCBuildAR project, the principles from the $3 \mathrm{C}$ model and the potential of mobile AR cards are now being brought together. How this can look like is now described by means of each $\mathrm{C}$.

\subsection{Content and AR}

Each PCBuildAR card shows a component of a classic computer system as a 2D representation. Currently, eight cards for eight different components and several variations of the components are produced (see Fig. 1). Variations are needed because later tasks require the exchange of components to solve a problem, e.g. upgrading a classic home computer for professional photo or video editing. Using a mobile device, tablet 
or smartphone, these images can be scanned with the camera and the additional information stored on them becomes visible. Declarative knowledge, e.g. the function, is conveyed for each component that is to be used afterwards (see Sect. 4.2). Because of the definition in [7,8], AR cannot be considered as a single medium but as multimedia. Therefore, learning materials with AR have to take into account the principles from the Cognitive Theory of Multimedia Learning (CTML), so that learners are not cognitively overstrained, but are supported in their learning processes [8]. For our AR cards, this means that $3 \mathrm{D}$ visualizations are not supplemented by written texts but by auditory information according to the modality principle. We also dispense with redundant representations by assigning AR a clear function, namely the extension of the physical card, which remains visible and thus relevant [9].

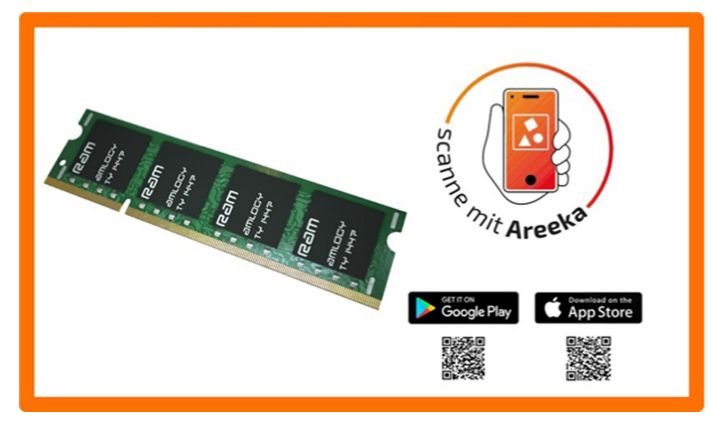

Fig. 1. Example of an AR card that visualizes the RAM memory component.

\subsection{Construction and AR}

Within the PCBuildAR project, experienced computer science teachers formulate the problem-based tasks according to technical issues. Examples include upgrading the computer so that professional video editing software can run without crashes or preparing the computer for graphically demanding computer games. With the latter task we also want to consider real problems from the current living environment of many children and young people. These tasks can be solved by first folding the cards correctly and then replacing the corresponding component. Afterwards the cards are scanned with a mobile device and the learners get feedback on the display if an appropriate solution for the problem has been found.

AR also serves here as a support system, as the information on the components can be made visible through scanning the markers, if needed. Especially beginners can benefit from such support actions as just-in-time information [10, 11].

\subsection{Communication and AR}

The communication component of our AR card is also linked to the tasks. If the cards are used in cooperative learning settings, the exchange between learners takes place. In the classroom, the teacher can also be called upon to provide support if necessary. 
The tasks that are to be solved in a team require the exchange of information between the learners. Basically, this component should be able to be implemented according to the ideas or conceptions of the respective teachers. We do not want to impose too many restrictions here (see Fig. 2).

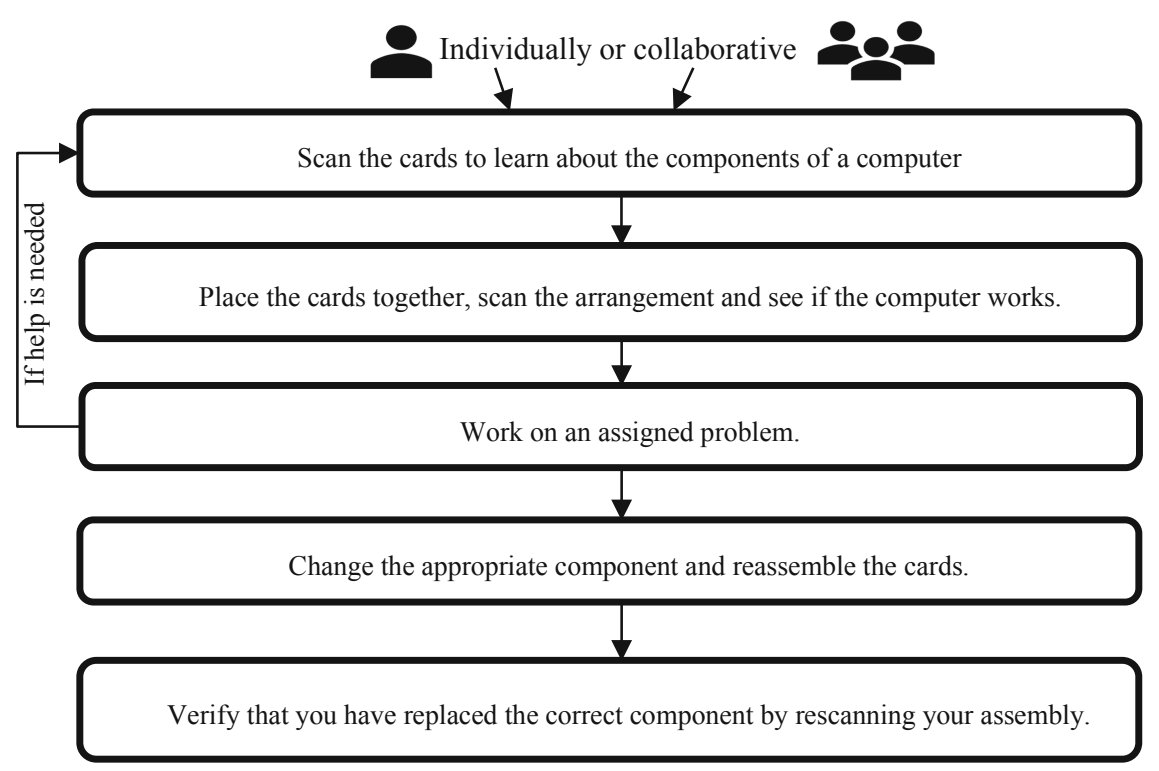

Fig. 2. Overview of the learning process with the AR cards.

\section{Conclusion and Outlook}

In this article we have described our AR cards for learning the components of computer systems, created in the PCBuildAR project. The $3 \mathrm{C}$ model serves as instructional approach. Accordingly, the contents to be taught are designed according to the CTML and the declarative knowledge is subsequently applied via problem solving tasks. Learning can be done individually or in cooperation with others. The communication component is not fixed but can be implemented according to the learning objectives and the teacher's own ideas.

The next step for us is to test the PCBuildAR cards empirically in schools and to investigate to what extent the combination of $3 \mathrm{C}$ and $\mathrm{AR}$ can help learners build knowledge structures and supports the application of this knowledge in the field of technical problem solving in computer science lessons. The cards are then made available as open educational resources (OER) for further use.

Funding. The PCBuildAR project is supported by the Innovation Foundation for Education and the Austrian Exchange Service OeAD. 


\section{References}

1. Informatics Europe \& ACM Europe Working Group: Informatics education: Europe cannot afford to miss the boat (2013)

2. Kerres, M., Witt, C.D.: A didactical framework for the design of blended learning arrangements. J. Educ. Media 28, 101-113 (2003). https://doi.org/10.1080/1358165032000165653

3. El Sayed, N.A.M., Zayed, H.H., Sharawy, M.I.: ARSC: augmented reality student card - an augmented reality solution for the education field. Comput. Educ. 56, 1045-1061 (2011). https://doi.org/10.1016/j.compedu.2010.10.019

4. Chen, R.W., Chan, K.K.: Using augmented reality flashcards to learn vocabulary in early childhood education. J. Educ. Comput. Res. 57, 1812-1831 (2019). https://doi.org/10.1177/ 0735633119854028

5. Giraudeau, P., et al.: CARDS: a mixed-reality system for collaborative learning at school. In: Proceedings of the 2019 ACM International Conference on Interactive Surfaces and Spaces, pp. 55-64. ACM, Daejeon (2019). https://doi.org/10.1145/3343055.3359721

6. Gardeli, A., Vosinakis, S.: ARQuest: a tangible augmented reality approach to developing computational thinking skills. In: 2019 11th International Conference on Virtual Worlds and Games for Serious Applications (VS-Games), pp. 1-8. IEEE, Vienna (2019). https://doi.org/ 10.1109/VS-Games.2019.8864603

7. Azuma, R., Baillot, Y., Behringer, R., Feiner, S., Julier, S., MacIntyre, B.: Recent advances in augmented reality. IEEE Comput. Graph. Appl. 21, 34-47 (2001). https://doi.org/10.1109/ 38.963459

8. Mayer, R.E.: Thirty years of research on online learning. Appl. Cogn. Psychol. 33, 152-159 (2019). https://doi.org/10.1002/acp.3482

9. Buchner, J., Zumbach, J.: Augmented reality in teacher education: a framework to support teachers' technological pedagogical content knowledge. Ital. J. Educ. Technol. 28(2) (2020). https://doi.org/10.17471/2499-4324/1151

10. Van Merrienboer, J.J.G., Kester, L.: The four-component instructional design model: multimedia principles in environments for complex learning. In: Mayer, R.E. (ed.) The Cambridge Handbook of Multimedia Learning, pp. 104-150. Cambridge University Press, Cambridge (2014)

11. Hmelo-Silver, C.E., Duncan, R.G., Chinn, C.A.: Scaffolding and achievement in problembased and inquiry learning: a response to Kirschner, Sweller, and Clark (2006). Educ. Psychol. 42, 99-107 (2007). https://doi.org/10.1080/00461520701263368

Open Access This chapter is licensed under the terms of the Creative Commons Attribution 4.0 International License (http://creativecommons.org/licenses/by/4.0/), which permits use, sharing, adaptation, distribution and reproduction in any medium or format, as long as you give appropriate credit to the original author(s) and the source, provide a link to the Creative Commons licence and indicate if changes were made.

The images or other third party material in this chapter are included in the chapter's Creative Commons licence, unless indicated otherwise in a credit line to the material. If material is not included in the chapter's Creative Commons licence and your intended use is not permitted by statutory regulation or exceeds the permitted use, you will need to obtain permission directly from the copyright holder.

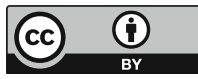

